\title{
CONVECTIVE INSTABILITY IN DIFFERENTIALLY ROTATING DISKS
}

\author{
DONGSU RYU AND JEREMY GOODMAN \\ Princeton University Observatory, Peyton Hall, Princeton, NJ 08544 \\ Received 1991 August 8; accepted 1991 September 18
}

\begin{abstract}
We perform a normal mode analysis for nonaxisymmetric perturbations in a thin, differentially rotating disk with a vertical structure that is isothermal and convectively unstable. The vertical gravity is assumed to be external and constant. The perturbation scale is assumed to be much shorter than the radius of the disk but comparable to or less than the thickness. The initial value problem is formulated in shearing coordinates. Dispersion relations are obtained for the three limiting cases of zero shear, axisymmetric perturbations, and small radial wavelengths. The full effects of shear are studied by integrating numerically the initial value problem. The main results of the paper are as follows: (1) Nonaxisymmetric local Fourier modes have a radial wavenumber that increases linearly with time in proportion to the shear times the azimuthal wavenumber. (2) While Coriolis forces exert stabilizing effects on the convective modes, reducing their growth rate and the range of unstable wavelengths, shear has destablizing effects inasmuch as it reduces the epicyclic frequency at a given angular velocity. (3) In a Keplerian disk, perturbations with azimuthal wavelengths about 2 times smaller than vertical wavelengths grow exponentially. Otherwise, perturbations do not grow until radial wavelengths become several times smaller than vertical wavelengths but grow exponentially after that. The exponential growth rate is roughly the limiting growth rate-the absolute value of the imaginary Brunt-Väisälä frequency - at infinitely small radial wavelengths. (4) A dispersion relation which can be used to estimate the instantaneous growth rates, or at least, the power bound of the instantaneous growth rates for nonaxisymmetric perturbations in differentially rotating disks is presented. (5) The angular momentum flux of the linear modes is nonzero only for nonaxisymmetric disturbances, and for these the flux is predominantly inward, i.e., in the direction of increasing angular velocity.
\end{abstract}

Subject headings: accretion, accretion disks - convection - hydrodynamics - instabilities

\section{INTRODUCTION}

Accretion disks are believed to play an important role in many astrophysical systems including binary systems, active galactic nuclei, and quasars. The evolution of such accretion disks is governed by the transport of angular momentum outward, allowing mass to flow inward. Because the mechanism is not well understood, it is conventional to assume that the rate of angular momentum transport depends on an effective viscosity of the form

$$
v=\frac{\alpha L^{2}}{\Omega},
$$

where $L$ and $\Omega$ are the disk thickness and the angular velocity and are functions of radius (Shakura \& Sunyaev 1973; Lynden-Bell \& Pringle 1974; Pringle 1981). The parameter $\alpha$ is dimensionless and presumed to be less than unity on physical grounds.

In disks of high opacity, very large temperature gradients may be necessary for radiative transport of energy from the disk midpoint to surface. Superadiabatic temperature gradients may arise and lead to convection. Lin \& Papaloizou (1980) have suggested that high opacities in the primordial solar nebula led to superadiabatic temperature gradients, convection, and hence turbulent viscosity. (See Cameron [1978a, b] for the discussion of other mechanisms to generate turbulent viscosity in the primordial solar nebula.) Motivated by these ideas, Ruden, Papaloizou, \& Lin (1988, henceforth RPL) analyzed the linear stability of axisymmetric convective modes in a polytropic disk with a superadiabatic vertical structure. They concluded that the growth rates (1) are smaller in a rotat- ing disk than in a nonrotating atmosphere of the same vertical structure; (2) increase as the square root of the radial wavenumber in their polytropic equilibria; (3) are bounded more generally by the maximum absolute value of the (imaginary) Brunt-Väisälä frequency (which tends to infinity at the surface of their models); and (4) are reduced at short wavelengths by thermal conduction. They also estimated $\alpha \sim 10^{-2}$ to $10^{-3}$ from the wavelengths and growth rates of the most favorable modes.

As noted by RPL, however, axisymmetric motions cannot exchange angular momentum between inviscid fluid elements. ${ }^{1}$ Therefore, angular-momentum transport by convection requires either secondary instabilities of the axisymmetric modes when they reach finite amplitudes, or nonaxisymmetric modes.

In this paper, therefore, we consider nonaxisymmetric convective modes in a differentially rotating disk. Shear greatly complicates the analysis of the nonaxisymmetric modes, so we carry out our analysis for an especially simple equilibrium with constant vertical scale height and constant gravitational field, but also with horizontal shear and rotation. In $\S 2$, the equilibrium disk structure is defined and the perturbation equations are derived using Lagrangian variables in shearing coordinates. In $\S 3$, the linearized equations are solved analytically in limiting cases or numerically otherwise to get the convective growth rates. The results are summarized and their implications discussed in $\S 4$. Certain mathematical details are presented in the Appendices.

\footnotetext{
${ }^{1}$ Unless the fluid is magnetized; see Balbus \& Hawley (1991) and Hawley \&
} Balbus (1991). 


\section{BASIC EQUATIONS}

We consider a fluid in a thin, differentially rotating disk which is not self-gravitating but is subject to the gravitational potential of a central object of mass $M$ located at $r=z=0$. The hydrodynamics of this fluid are described by the following equations:

$$
\partial_{t} \rho+\nabla \cdot(\rho u)=0
$$

$\partial_{t} u+u \cdot \nabla u+\frac{1}{\rho} \nabla p+2 \Omega \hat{z} \times u$

$$
\begin{gathered}
-\nabla\left(\frac{1}{2} \Omega^{2} r^{2}\right)+\nabla \phi(r, z)=0, \\
\partial_{t} p+u \cdot \nabla p+\gamma p \nabla \cdot u=0,
\end{gathered}
$$

where $\phi(r, z)$ is the external potential,

$$
\phi(r, z)=-\frac{G M}{\sqrt{r^{2}+z^{2}}} .
$$

The above equations are written in a frame rotating around $z$-axis with angular velocity $\Omega$, where $\Omega$ is the angular velocity of a circular orbit at $r=r_{0}$ and $z=0$, i.e.,

$$
\Omega=\sqrt{\frac{G M}{r_{0}^{3}}} .
$$

Since we are interested in the local dynamics of the fluid, we define a pseudo-Cartesian coordinate system extending over a small part of the disk comparable in horizontal scale with the disk thickness :

$$
\begin{gathered}
x \equiv r-r_{0}, \\
y \equiv r_{0}\left(\theta-\theta_{0}\right), \\
v_{x} \equiv u_{r}, \\
v_{y} \equiv \frac{r_{0}}{r}\left(u_{\theta}-\Omega r\right), \\
v_{z} \equiv u_{z} .
\end{gathered}
$$

We consider that

$$
\frac{x}{r_{0}} \sim \frac{y}{r_{0}} \sim \frac{z}{r_{0}} \sim \epsilon \ll 1,
$$

and also

$$
\frac{v_{x}}{\Omega r_{0}} \sim \frac{v_{y}}{\Omega r_{0}} \sim \frac{v_{z}}{\Omega r_{0}} \sim \epsilon \ll 1,
$$

where $\epsilon \equiv L / r_{0}$ and $L$ is the thickness of the disk. In the spirit of the local approximation, we expand equations (2.1a)-(2.1d) with respect to $\epsilon$ and keep only the lowest nonzero power of $\epsilon$. The resulting shearing sheet equations are

$$
\begin{gathered}
\partial_{t} \rho+\nabla \cdot(\rho v)=0 \\
\partial_{t} v+v \cdot \nabla v+\frac{1}{\rho} \nabla p+2 \Omega \hat{z} \times v+4 A \Omega x \hat{x}+\Omega^{2} z \hat{z}=0 \\
\partial_{t} p+v \cdot \nabla p+\gamma p \nabla \cdot v=0
\end{gathered}
$$

where $A$ is the Oort constant given by

$$
A=\left.\frac{r}{2} \frac{d \Omega}{d r}\right|_{r_{0}}
$$

and is assumed to be independent of $x$. (Note that this differs by a sign from the conventional first Oort constant of galactic dynamics see Mihalas \& Binney 1981.) The unperturbed velocity is

$$
\boldsymbol{v}_{0}=2 A x \hat{y} .
$$

The above model is commonly called the shearing sheet. The shearing-sheet model has been used in many previous investigations of local disk physics, notably Goldreich \& Lynden-Bell (1965), Julian \& Toomre (1966), and Shu (1974).

In the following sections, we replace the vertical gravity $\Omega^{2} z$ by a parameter $g$ that is constant apart from a change in sign at the midplane of the disk. We suppose that $g$ represents, for instance, the density-weighted root mean square average of $\Omega^{2} z$. Although this idealization takes us one step further from realistic disks, it enormously simplifies the mathematical analysis by permitting Fourier analysis in the vertical as well as the horizontal directions (see below). Nonaxisymmetric modes with shear can then be described by ordinary differential equations in time $(t)$ rather than partial differential equations in $t$ and $z$. We believe that the important physical effects of shear and rotation on the convective modes are accessible within this constant- $g$ model.

In a Keplerian disk with angular velocity (2.2), we have

$$
A=-\frac{3}{4} \Omega \text {. }
$$

It will be useful, however, occasionally to consider the case of a uniformly rotating disk, $A=0$, so we shall retain $A$ as an independent parameter.

\subsection{Equilibrium Disk Structure}

For simplicity, we choose an equilibrium disk which is locally isothermal with constant adiabatic sound speed,

$$
c_{s}=\sqrt{\gamma \frac{p}{\rho}} .
$$

In a constant gravitational field, vertical hydrostatic equilibrium,

$$
\frac{1}{\rho} \partial_{z} p+g=0
$$

implies a constant density and pressure scale height, $L$ :

$$
\begin{aligned}
& \rho(z)=\rho(0) \exp \left(-\frac{|z|}{L}\right), \\
& p(z)=p(0) \exp \left(-\frac{|z|}{L}\right),
\end{aligned}
$$

where

$$
L=\frac{c_{s}^{2}}{\gamma|g|} .
$$

On the other hand, had we taken a linearly increasing gravitational field, $g \rightarrow \Omega^{2} z$, we would have found $\rho(z)=\rho(0) \exp$ 
$\left(-\gamma \Omega^{2} z^{2} / 2 c_{s}^{2}\right)$; so if our constant $g^{2}$ is to be identified with the density-weighted average of $\left(\Omega^{2} z\right)^{2}$, then

$$
g=\Omega \frac{c_{s}}{\sqrt{\gamma}} \operatorname{sign}(z)=\Omega^{2} L \operatorname{sign}(z) .
$$

This equilibrium is unstable to convection if $\gamma<1$ (see, e.g., Shu 1974). A virtual adiabatic and isobaric displacement of a fluid element through a vertical distance $\Delta z$ produces a vertical acceleration $\Delta a_{z}=-N_{\mathrm{B}}^{2} \Delta z$, where the Brunt-Väisälä frequency $N_{\mathbf{B}}$ is defined through

$$
N_{\mathbf{B}}^{2}=g\left(\frac{1}{c_{s}^{2} \rho} \frac{d p}{d z}-\frac{1}{\rho} \frac{d \rho}{d z}\right)=\frac{\gamma-1}{\gamma} \Omega^{2} .
$$

Such displacements are unstable if $N_{\mathrm{B}}^{2}<0$.

An adiabatic exponent $\gamma<1$ makes little thermodynamic sense. For example, it implies heat capacities $C_{p}<C_{V}$ so that $p d V$ work contributes to the internal energy with the wrong sign. There are, however, no difficulties with the purely hydrodynamic equations (2.1a)-(2.1d) and (2.5a)-(2.5d). Indeed, the hydrodynamic effects of $\gamma<1$ could be physically approximated by an appropriate choice of heating and cooling functions and a thermal time scale much shorter than the hydrodynamic one. For example, a constant heating rate per unit mass and a cooling rate proportional to $\rho^{a} T^{b}$ yield an effective adiabatic index $\gamma=1-(a / b)$. We are in any case willing to pay the price of an unphysical $\gamma$ in order to be able to study convection in a medium of constant $g$ and $L$.

\subsection{Perturbation Equations}

We choose to linearize equations (2.5a) $-(2.5 \mathrm{~d})$ in Lagrangian form. The Lagrangian equation of motion is

$$
D_{t}^{2} x=f(x, t),
$$

where $x$ is the location of the fluid element at time $t, D_{t}$ is the Lagrangian time derivative following the fluid element, and $f$ is the acceleration at point $(x, t)$ given by

$$
f(x, t)=-\frac{1}{\rho} \nabla p-2 \Omega \times D_{t} x-4 A \Omega x \hat{x}-g \hat{z} .
$$

In the perturbed state, let $\xi\left[x^{(o)}, t\right]$ be the (infinitesimal) displacement of a fluid element from its unperturbed position, $\boldsymbol{x}^{(o)}$; that is,

$$
\boldsymbol{x}=\boldsymbol{x}^{(o)}+\xi .
$$

Let $\delta f\left[x^{(o)}, t\right]$ be the perturbed acceleration at the unperturbed position, i.e., the Eulerian perturbation of the acceleration. Then, the linearized perturbation equation is

$$
D_{t}^{2} \xi=\delta f\left[x^{(o)}, t\right]+\left[\xi \cdot \nabla^{(o)}\right] f^{(o)}\left[x^{(o)}, t\right] .
$$

The second term on the right-hand side is due to the change of the position in the unperturbed force field. Since in equilibrium

$$
f^{(o)}\left[x^{(o)}, t\right]=0,
$$

the second term on the right-hand side of equation (2.16) vanishes.
By collecting terms and workng on each Cartesian component separately we find

$$
\begin{gathered}
D_{t}^{2} \xi_{x}=2 \Omega D_{t} \xi_{y}-4 A \Omega \xi_{x}-\frac{1}{\rho} \partial_{x} \delta p \\
D_{t}^{2} \xi_{y}=-2 \Omega D_{t} \xi_{x}-\frac{1}{\rho} \partial_{y} \delta p \\
D_{t}^{2} \xi_{z}=-g \frac{\delta \rho}{\rho}-\frac{1}{\rho} \partial_{z} \delta p
\end{gathered}
$$

where

$$
\delta \rho=-\rho \nabla \cdot \xi-\xi_{z} \frac{d \rho}{d z}
$$

and

$$
\delta p=-\rho c_{s}^{2} \nabla \cdot \xi+\rho g \xi_{z},
$$

Note that, since $\xi$ in the above equations is first order in perturbation, the Lagrangian time derivative can be replaced with its zeroth-order form, i.e., the derivative along the unperturbed flow,

$$
D_{t} \rightarrow \partial_{t}+2 A x \partial y \text {. }
$$

Since equations (2.18a)-(2.18e) do not depend explicitly on $y$, they may be Fourier decomposed in $y$. Because $D_{t}$ contains an explicit factor of $x$, however, the equations cannot be Fourier decomposed in $x$ as they stand. ${ }^{2}$ The dependence on $x$ can be removed by using shearing coordinates defined by (see Goldreich \& Lynden-Bell 1965)

$$
\begin{gathered}
t^{\prime}=t, \\
x^{\prime}=x, \\
y^{\prime}=y-2 A x t, \\
z^{\prime}=z ;
\end{gathered}
$$

whence

$$
\begin{gathered}
D_{t}=\partial_{t}^{\prime}, \\
\partial_{x}=\partial_{x}^{\prime}-2 A t^{\prime} \partial_{y}^{\prime}, \\
\partial_{y}=\partial_{y}^{\prime}, \\
\partial_{z}=\partial_{z}^{\prime} .
\end{gathered}
$$

We assume $\xi$ of the form

$$
\xi\left(x^{\prime}, y^{\prime}, z^{\prime}, t^{\prime}\right)=\xi\left(t^{\prime}\right) \exp \left(\frac{\left|z^{\prime}\right|}{2 L}\right) \exp \left(i k_{x} x^{\prime}+i k_{y} y^{\prime}+i k_{z} z^{\prime}\right)
$$

(The justification for the exponential factor involving $\left|z^{\prime}\right| / 2 L$ will be given in Appendix A.) With the above form, the energy associated with perturbations, which is proportional to $\rho \dot{\xi}^{2}$, is spatially constant when it is averaged over perturbation wavelengths. The perturbation equations in shearing coordinates

\footnotetext{
${ }^{2}$ Actually, they can be simultaneously transformed in $x$ and $y$, but the factor of $x$ transforms to $i \partial_{k}$, so $D_{t} \rightarrow \partial_{t}-2 A k_{y} \partial_{k}$. Since there are no other derivatives in Fourier space, perturbations propagate along the characteristics $k_{x}(t)=k_{x}(0)-2 A k_{y} t$.
} 
become, for $z^{\prime}>0$ (we omit the prime on $t$ ),

$$
\begin{gathered}
\partial_{t}^{2} \xi_{x}=2 \Omega \partial_{t} \xi_{y}-4 A \Omega \xi_{x}-\frac{1}{\rho} i k_{x} \delta p \\
\partial_{t}^{2} \xi_{y}=-2 \Omega \partial_{t} \xi_{x}-\frac{1}{\rho} i k_{y} \delta p \\
\partial_{t}^{2} \xi_{z}=-g \frac{\delta \rho}{\rho}-\frac{1}{\rho}\left(i k_{z}+\frac{1}{2 L}\right) \delta p \\
\frac{\delta \rho}{\rho}=-i k \cdot \xi+\frac{1}{2 L} \xi_{z} \\
\frac{\delta p}{\rho}=-c_{s}^{2} i k \cdot \xi-\left(\frac{c_{s}^{2}}{2 L}-g\right) \xi_{z}
\end{gathered}
$$

Here,

$$
k_{x}(t) \equiv k_{x}-2 A k_{y} t,
$$

and $\boldsymbol{k} \equiv\left[k_{x}(t), k_{y}, k_{z}\right]$. The corresponding equations for $z^{\prime}<0$ can be obtained by replacing $L$ with $-L$. At $z^{\prime}=0$, due to the assumed discontinuity in $g$ and consequently in $\partial_{z} \rho$ and $\partial_{z} p$, the equations are not well defined.

Equations (2.23a)-(2.23e) are ordinary differential equations in time (with $k_{x}, k_{y}$, and $k_{z}$ as constant parameters) and constitute an initial value problem. The transformation to shearing coordinates has introduced an explicit time-dependence, here expressed through $k_{x}(t)$, which the original equations (2.18) lack. One may ask why we do not work with equations (2.18) and Fourier transform in $t$ and $y$ rather than $x$ and $y$. The answer is that we suspect that there are no nonaxisymmetric local modes of fixed spatial planform in nonshearing coordinates. Disturbances of the form (2.22) are after all spatially complete, and equations (2.23) and (2.24) show that nonaxisymmetric (i.e., $k_{y} \neq 0$ ) linear superpositions of such disturbances are inexorably carried toward increasing $k_{x}(t) / k_{y}$; a constant spatial planform therefore requires a Fourier transform containing a supply of infinitely tightly wrapped leading waves at $k_{x} / k_{y}=$ $-\infty$. A function with such a Fourier transform cannot be differentiable in $x$, and in any case any viscosity at all would suppress the large $-k_{x}$ components. Boundaries at finite $x$ would change this situation since they reflect trailing $\left(k_{x} / k_{y}>\right.$ 0 ) waves back to leading ones; however, disturbances depending on such boundaries are not truly local modes.

\subsection{Vertical Boundary Conditions}

Following previous work (see, e.g., Parker 1966 and Shu 1974), we require that the fluid does not cross the midplane of the disk, i.e.,

$$
\xi_{z}=0 \text { at } z=0 .
$$

This is really the only vertical boundary condition compatible with equations (2.18) or (2.23) because of the discontinuity in $g$ at $z=0$. Unfortunately, it permits only solutions that are symmetric across the midplane (i.e., symmetric in $\delta \rho$ and $\delta p$ but antisymmetric in $\xi_{z}$ ). We note that RPL found the fastest growing axisymmetric mode at each radial wavenumber to be antisymmetric in $z$.

In limiting cases where $k_{x}(t)$ is effectively time-independent so that an algebraic dispersion relation exists $(\S 3.1,2,3)$, the above boundary condition is satisfied if the same growth rate $s$ remains as an eigenvalue of the equations when $k_{z}$ is replaced by $-k_{z}$. Otherwise $(\S 3.4)$, if $\xi_{z}\left(t ; k_{x}, k_{y}, k_{z}\right) / \xi_{z}\left(t ; k_{x}, k_{y},-k_{z}\right)$, or or equivalently $\operatorname{Re}\left[\xi_{z}\left(t ; k_{x}, k_{y}, k_{z}\right)\right] / \operatorname{Im}\left[\xi_{z}\left(t ; k_{x}, k_{y}, k_{z}\right)\right]$, is independent of time, symmetric and antisymmetric solutions can be decoupled and the above condition can be satisfied by taking symmetric combinations. All the cases considered in $\S 3$ do in fact permit such symmetric solutions.

\section{ANALYSIS OF EIGENMODES}

In the first three subsections, we consider the limiting cases where true normal mode solutions with $\xi(t) \propto \exp (s t)$ exist, and dispersion relations can be derived. These cases were already considered by Shu (1974) and our dispersion relations reduce to his. In Appendix B, we show that, in these limiting cases, $s^{2}$ is real and thus overstability does not occur. The growth rate $s$ may, however, be purely imaginary, in which case the corresponding mode is purely oscillatory without growth or decay.

\subsection{Rigidly Rotating Disks $(A=0)$}

From the perturbation equations (2.23), we get

$$
\begin{aligned}
s^{2}\left\{s^{4}+\left(c_{s}^{2} k_{L}^{2}\right.\right. & \left.+\kappa^{2}\right) s^{2}+c_{s}^{2} \\
\times & {\left.\left[N_{\mathrm{B}}^{2}\left(k_{x}^{2}+k_{y}^{2}\right)+\kappa^{2}\left(k_{z}^{2}+\frac{1}{4 L^{2}}\right)\right]\right\}=0, }
\end{aligned}
$$

where

$$
k_{L}^{2} \equiv k_{x}^{2}+k_{y}^{2}+k_{z}^{2}+\frac{1}{4 L^{2}}
$$

and

$$
\kappa^{2} \equiv 4 \Omega(\Omega+A)=\left.\frac{1}{r^{3}} \frac{d}{d r}\left[r^{2} \Omega(r)\right]^{2}\right|_{r=r_{0}} .
$$

Thus $\kappa$ is the epicyclic frequency; for $A=0, \kappa \rightarrow 2 \Omega$, but we have introduced the general expression here for future use (§ 3.2).

The overall factor of $s^{2}$ in this dispersion relation signifies the existence of two trivial modes in which the perturbation is just an infinitesimal relabelling of the fluid elements without any change in the Eulerian variables.

The only potentially negative term in the dispersion relation is the one proportional to $N_{\mathbf{B}}^{2}$. The dispersion relation is negative at $s=0$, and hence there exists an unstable mode with $s^{2}>0$, if and only if

$$
N_{\mathrm{B}}^{2}<-\kappa^{2}\left[\frac{1+4 k_{z}^{2} L^{2}}{4\left(k_{x}^{2}+k_{y}^{2}\right) L^{2}}\right] .
$$

Because of the final form of $N_{B}^{2}$ given in equation (2.13), the relation (3.2) confirms that our isothermal model is convectively unstable only if $\gamma-1<0$. We have avoided explicit use of $\gamma$ in equations (3.1) and (3.2) because these relations can be extended to more general atmospheres in the WKB limit, provided $N_{\mathrm{B}}^{2}$ is appropriately redefined using the first form given in equation (2.13).

We see also from equation (3.2) that rotation has a stabilizing influence at short vertical wavelengths and long horizontal wavelengths. [It is only by considering the case of axisymmetric modes with shear, however, that we see that the critical rotational parameter is really the epicyclic frequency $\kappa$ rather than the Coriolis parameter $\Omega$ itself $(\S 3.2)$. 

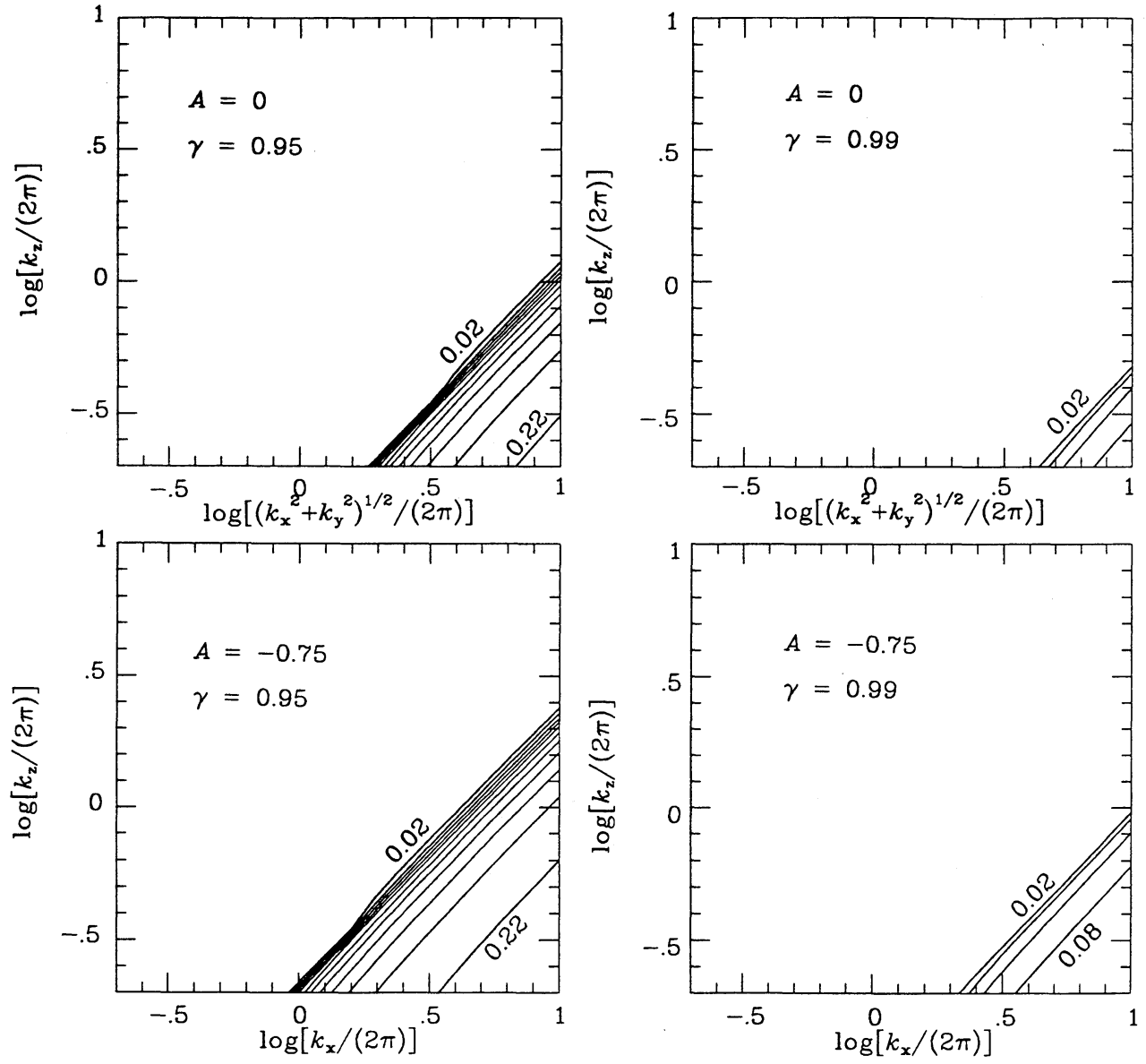

Fig. 1.-Contours of equal growth rate, $s$, in units of the angular velocity $\Omega$. Interval of contours is $0.02 \Omega$. The upper two plots show the growth rates of the perturbations in rigidly rotating disks $(A=0)$ with $\gamma=0.95$ and 0.99 [or $\left(-N_{\mathrm{B}}^{2}\right)^{1 / 2}=0.2294 \Omega$ and $0.1005 \Omega$ ]. The lower two plots show the growth rates of the axisymmetric perturbations in a Keplerian disk $(A=-0.75 \Omega)$ with $\gamma=0.95$ and 0.99 . The modified wavenumber $k / 2 \pi$ is given in units of the reciprocal of the disk scale height, $1 / L$.

The upper two plots in Figure 1 show contours of equal growth rate $s$ in units of $\Omega$ for two different values of $\gamma, 0.95$ and 0.99. For this purpose [though not in deriving eq. (3.1)] we assume that $g$ is given by equation (2.12), hence the corresponding values of

$$
\sqrt{-N_{\mathbf{B}}^{2}} \rightarrow \Omega \sqrt{(1-\gamma) / \gamma}
$$

are $0.2294 \Omega$ and $0.1005 \Omega$, respectively. For For $\gamma=0.95$, unstable modes exist for

$$
\sqrt{k_{x}^{2}+k_{y}^{2}} \gtrsim 8 k_{z}
$$

and the growth rate approaches $\left(-N_{\mathbf{B}}^{2}\right)^{1 / 2}$, the maximum allowed value (see $\S 3.3$ ), for

$$
\sqrt{k_{x}^{2}+k_{y}^{2}} \gtrsim 40 k_{z} \text {. }
$$

For $\gamma=0.99$, unstable modes exist for

$$
\sqrt{k_{x}^{2}+k_{y}^{2}} \gtrsim 20 k_{z}
$$

and the growth rate approaches $\left(-N_{\mathrm{B}}^{2}\right)^{1 / 2}$ for $\sqrt{k_{x}^{2}+k_{y}^{2}} \gtrsim$ $50 k_{z}$.

$$
\text { 3.2. Axisymmetric Modes }\left(k_{y}=0\right)
$$

In this case, equation (2.24) implies that $k_{x}$ is constant despite the shear. The algebraic dispersion relation (3.1) and the stability criterion (3.2) apply to this case also, provided we take $k_{y}=0$ in those formulae.

An astrophysical disk with the usual sense of differential rotation $(A / \Omega<0$, so $|\Omega|$ decreases with radius) is clearly less stable than a rigidly rotating disk of the same angular velocity. Nevertheless, it remains more stable than a nonrotating atmosphere of the same vertical stratification provided $\kappa^{2}>0$. The Rayleigh centrifugal instability appears for $\kappa^{2}<0$, but this is of little interest to us since we are mainly concerned with the Keplerian case, $\kappa=\Omega$.

The lower two plots in Figure 1 were calculated in the same way as the upper two, but for $A=-3 \Omega / 4$ and $k_{y}=0$, the axisymmetric and Keplerian case. For $\gamma=0.95$, unstable modes exist for $k_{x} \gtrsim 4 k_{z}$ and the growth rate approaches ( $\left.-N_{\mathrm{B}}^{2}\right)^{1 / 2}$ for $k_{x} \gtrsim 20 k_{z}$. For $\gamma=0.99$, unstable modes exist for $k_{x} \gtrsim 10 k_{z}$ and the growth rate approaches $\left(-N_{\mathrm{B}}^{2}\right)^{1 / 2}$ for $k_{x} \gtrsim$ $30 k_{z}$.

\subsection{Limiting Case with Large Radial Wavenumbers $\left(k_{x} \rightarrow \infty\right)$}

Since $\dot{k}_{x}(t)=-2 A k_{y}$ (eq. [2.24]), nonaxisymmetric perturbations in differentially rotating disks will have eventually very large radial wavenumbers. Ultimately $k_{x}(t) \gg k_{y}$ and $\dot{k}_{x}(t) / k_{x}(t) \ll|\Omega|,|A|$, or $\left(-N_{\mathrm{B}}^{2}\right)^{1 / 2}$. In this limit we may expect 
that the time-dependent equations (2.23) for the amplitude of such perturbations should have WKB solutions of the form

$$
\xi(t)=X(t) \exp \left\{\int s\left[\boldsymbol{k}\left(t^{\prime}\right)\right] d t^{\prime}\right\},
$$

where $\boldsymbol{k}(t) \equiv\left[k_{x}(t), k_{y}, k_{z}\right]$ is the instantaneous wavenumber with $k_{x}(t) \equiv k_{x}(0)-2 A k_{y} t$ and $s[k(t)]$ is the corresponding instantaneous growth rate calculated from equation (3.1).

Hence, an important limiting case of the dispersion relation (3.1) is $k_{x} \gg k_{y}, k_{z}, 1 / L$. From the perturbation equations in equation (2.23), it follows that in this limit,

$$
k_{x} \xi_{x} \sim k_{y} \xi_{y} \sim k_{z} \xi_{z},
$$

whence the radial displacement is very small compared to the azimuthal and vertical displacements. The unstable roots of the dispersion relation reduce to

$$
s^{2}=-N_{\mathrm{B}}^{2}=\frac{1-\gamma}{\gamma} \Omega^{2} .
$$

Note that the same dispersion relation in the limit $\left(k_{x}^{2}\right.$ $\left.+k_{y}^{2}\right)^{1 / 2} \gg k_{z}, L$ for rigidly rotating disks $(A=0)$ and in the limit $k_{x} \gg k_{z}, 1 / L$ for axisymmetric perturbations $\left(k_{y}=0\right)$. Note further from the form of equation (3.1) that the growth rate can never be larger than equation (3.5) (because $s^{2}$ must be real, by Appendix B, and so all other terms but those which yield this relation are positive) in the two limiting cases considered in $\S 3.1 .2$. The limit $s \leq\left(-N_{\mathrm{B}}^{2}\right)^{1 / 2}$ is expected to hold for convective instabilities of disks in general, simply because buoyancy cannot accelerate fluid elements upwards faster than $|g|$ (see Chandrasekhar 1961).

\subsection{Differentially Rotating Disks}

In the general case of nonaxisymmetric perturbations in differentially rotating disks, no true normal mode exists and the linearized equations (2.23) must be integrated numerically. We have done this using the Runge-Kutta-Verner fifth and sixth order method. We arbitrarily chose the initial conditions

$$
\xi_{y}=1 \text { and } \xi_{x}=\xi_{z}=\partial_{t} \xi_{x}=\partial_{t} \xi_{y}=\partial_{t} \xi_{z}=0 .
$$

This produces adiabatic Eulerian perturbations corresponding to initial density and pressure perturbations $\delta \rho / \rho=-i k_{y}$ and $\delta p / p=-i \gamma k_{y}$. With the above initial conditions, $\xi_{x}, \xi_{y}, \partial_{t} \xi_{x}$, and $\partial_{t} \xi_{y}$ remain but $\xi_{z}$ and $\partial_{t} \xi_{z}$ become complex, as can be seen in the equations (2.23). Our results for the growth rates and eigenfunctions are insensitive to the choice of the initial conditions, as expected for exponentially growing disturbances.

Figure 2 shows an example of the evolution of a nonaxisymmetric perturbation in a Keplerian disk $(A=-0.75 \Omega)$ with $\gamma=0.95\left[\left(-N_{B}^{2}\right)^{1 / 2}=0.2294 \Omega\right]$. The vertical and azimuthal wavenumbers are $k_{z}=k_{y}=2 \pi / L$, and the radial wavenumber $k_{x}(t)$, is set to be zero at $t=0$. The integration starts from $t=-100 / \Omega$ and ends at $t=100 / \Omega$. After an initial period of adjustment the plots show approximately exponential growth except for a period of stagnation around $t \sim 0$, where $\left[k_{x}^{2}(t)+k_{y}^{2}\right]^{1 / 2}$ is smallest. The exponential growth rates estimated from the logarithmic time derivatives of $\xi_{x} / k_{x}, \xi_{y}$, or $\xi_{z}$ at large $|t|$ are close to the limiting growth rate, $0.2294 \Omega$. The duration of the stagnant period depends upon $k_{y} L, k_{z} L$, $\left(-N_{\mathrm{B}}^{2}\right)^{1 / 2} / \Omega$ (or equivalently $\gamma$, in our case), and no doubt upon $2 A / \Omega$, although we have considered only the Keplerian case.
Also in Figure 2, the dashed line plots the evolution of $\xi_{z}(t)$ calculated by

$$
\xi_{z}(t) \propto \exp \left[\int_{t} \operatorname{Re}\left\{s\left[k\left(t^{\prime}\right)\right]\right\} d t^{\prime}\right],
$$

where $s[\boldsymbol{k}(t)]$ is one of the roots of equation (3.1) with the instantaneous wavenumber $k(t) \equiv\left[k_{x}(t), k_{y}, k_{z}\right]$ and the Keplerian value for $\kappa^{2}$. Except around the stagnation period during which the estimated growth with the above formula is slightly less than the growth calculated by numerically integrating the equations (2.23) (see Table 2 and the discussion below), the dashed line follows closely the solid lines. This indicates that the dispersion relation in equation (3.1) can be used to estimate approximately the instantaneous growth rates for nonaxisymmetric perturbations in differentially rotating disks.

Figures 3 and 4 exhibit the evolution of the $z$-displacement, $\xi_{z}$, of the nonaxisymmetric perturbations for different $N_{\mathrm{B}}$ 's (or $\gamma$ 's) and vertical and azimuthal wavenumbers. One noticeable point, is that, for given $N_{\mathrm{B}}$, the exponential growth rate does not depend on $k_{y}$ and $k_{z}$ but the stagnation period does. For $\left(-N_{\mathrm{B}}^{2}\right)^{1 / 2}$ closer to zero, larger vertical wavenumbers, and smaller azimuthal wavenumbers, the period is longer. Hence, for given $N_{\mathrm{B}}$, the net amplification of the perturbation during our integration is therefore determined by the stagnation period. In order to quantify these results, we have estimated the averaged growth rates across the interval $-10 / \Omega \leq t \leq 10 /$ $\Omega$ in Table 1 . As expected, the averaged growth rate is larger if the stagnation period is smaller, or for larger $\left(-N_{B}^{2}\right)^{1 / 2}$ smaller vertical wavenumbers and larger azimuthal wavenumbers. For $\gamma=0.95$ and 0.99 , the averaged growth rate approaches the maximum allowed one if $k_{x} \gtrsim 5 k_{z}$ and $10 k_{z}$.

In order to compare the above time-averaged growth rates for nonaxisymmetric perturbations with those estimated from the dispersion relation in equation (3.1), we calculate the following quantity for different $N_{\mathbf{B}}$ 's and vertical and azimuthal wavenumbers,

$$
\int_{-10 / \Omega}^{10 / \Omega} \operatorname{Re}\{s[k(t)]\} d t /(20 / \Omega),
$$

where $s[\boldsymbol{k}(t)]$ is one of the roots of equation (3.1) with the instantaneous wavenumber $\boldsymbol{k}(t) \equiv\left[k_{x}(t), k_{y}, k_{z}\right]$. The resulting growth rates are given in Table 2. While for $k_{y} \sim k_{z}$ the growth rates in Table 2 are smaller (up to $\sim 2$ times) than those in Table 1 , for $k_{y} \gtrsim 2 k_{z}$ the growth rates become comparable. Although the difference in growth rates in Tables 1 and 2 depends on the time interval over which the growth rate is averaged, the result confirms that the dispersion relation in equation (3.1) can be used to estimate the instantaneous growth rates, or at least, the lower bound of the instantaneous growth rates for nonaxisymmetric perturbations in differentially rotating disks.

\section{DISCUSSION}

The inequality (3.2) confirms the well-known result that rotation has a stabilizing effect upon convective modes. Nevertheless, our models are always unstable at sufficiently large horizontal wavenumbers whenever $N_{\mathrm{B}}^{2}<0$. Inasmuch as we have seen that the nonaxisymmetric disturbances eventually [when $k_{x}(t) \gg k_{y}$ ] grow at essentially the same rate as axisymmetric modes of the same $k_{x}$, it follows that all nonaxisymmetric disturbances are ultimately unstable in our model. 

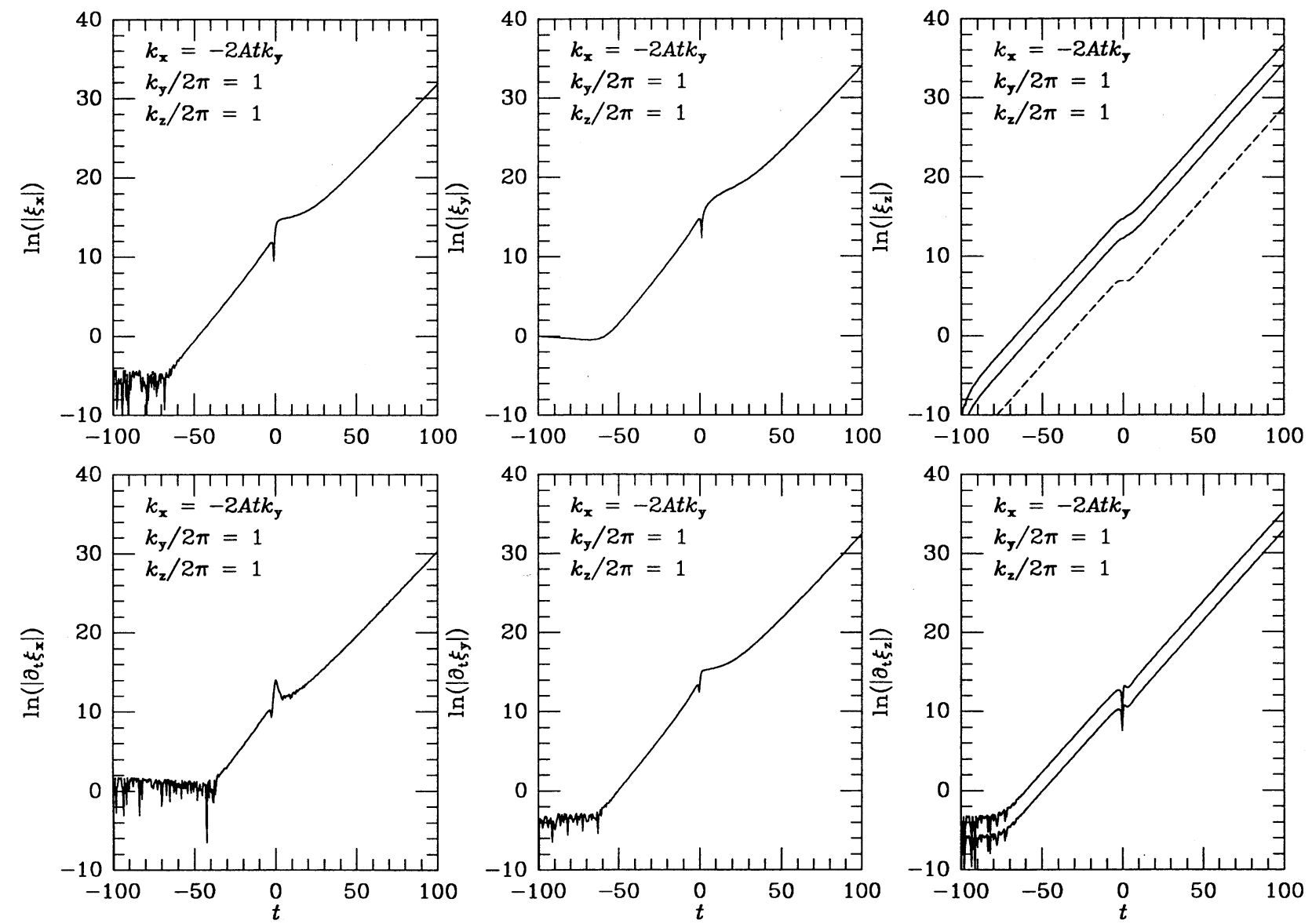

Fig. 2. Evolution of the nonaxisymmetric perturbation in a Keplerian disk $(A=-0.75 \Omega)$ with $\gamma=0.95$ [or $\left.\left(-N_{\mathrm{B}}^{2}\right)^{1 / 2}=0.2294 \Omega\right]$. Vertical and azimuthal wavelengths are equal to the disk scale height, $L$. Time $t$ is given in units of the reciprocal of the angular velocity, $1 / \Omega$ The initial conditions at $t=-100 / \Omega$ are $\xi_{y}=1$ and $\xi_{x}=\xi_{z}=\partial_{t} \xi_{x}=\partial_{t} \xi_{y}=\partial_{t} \xi_{z}=0$. While $\xi_{x}, \xi_{y}, \partial_{t} \xi_{x}$, and $\partial_{t} \xi_{y}$ remain real, $\xi_{z}$ and $\partial_{t} \xi_{z}$ become complex. The two solid lines in the $\xi_{z}$ and $\partial_{t} \xi_{z}$ plots correspond to real (upper line) and imaginary (lower line) parts, respectively. The dashed line in the $\xi_{z}$ plot shows the estimated evolution of $\xi_{z}$ using the dispersion relation (3.1) (see eq. [3.7]).

TABLE 1

Average Growth Rate IN UNITS OF $\Omega$ BeTWEen $-10 / \Omega \leq t \leq 10 / \Omega$ IN A KePleRIAN DisK ${ }^{\mathrm{a}}$

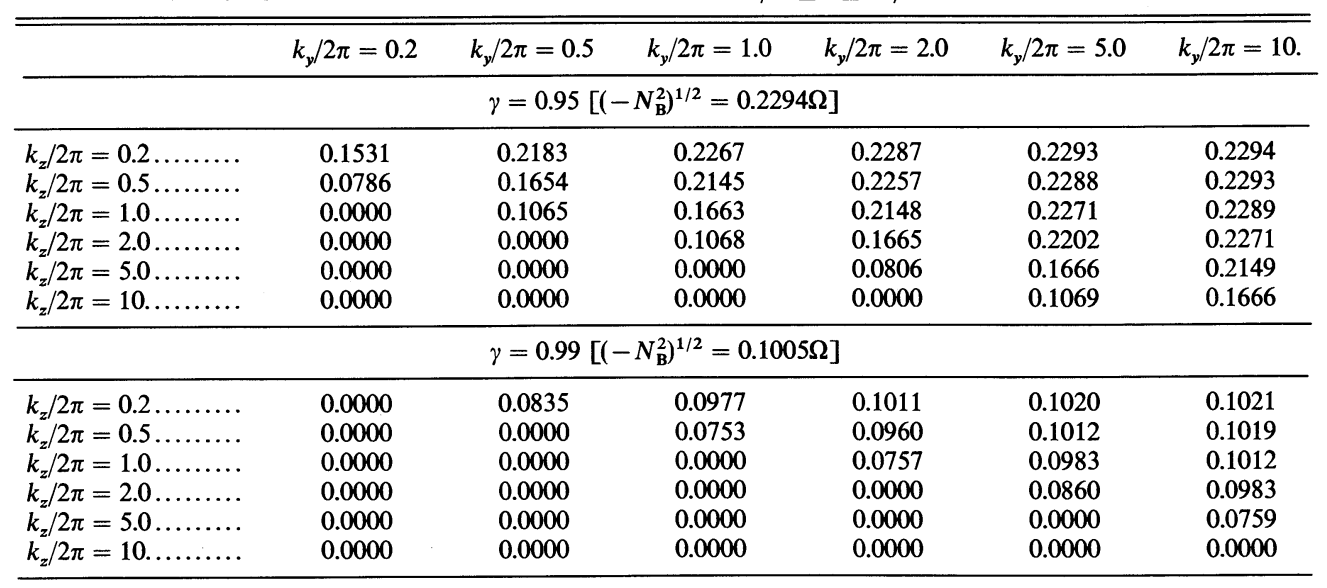

a Calculated by integrating numerically the linearized shearing sheet equations; see text for details. 

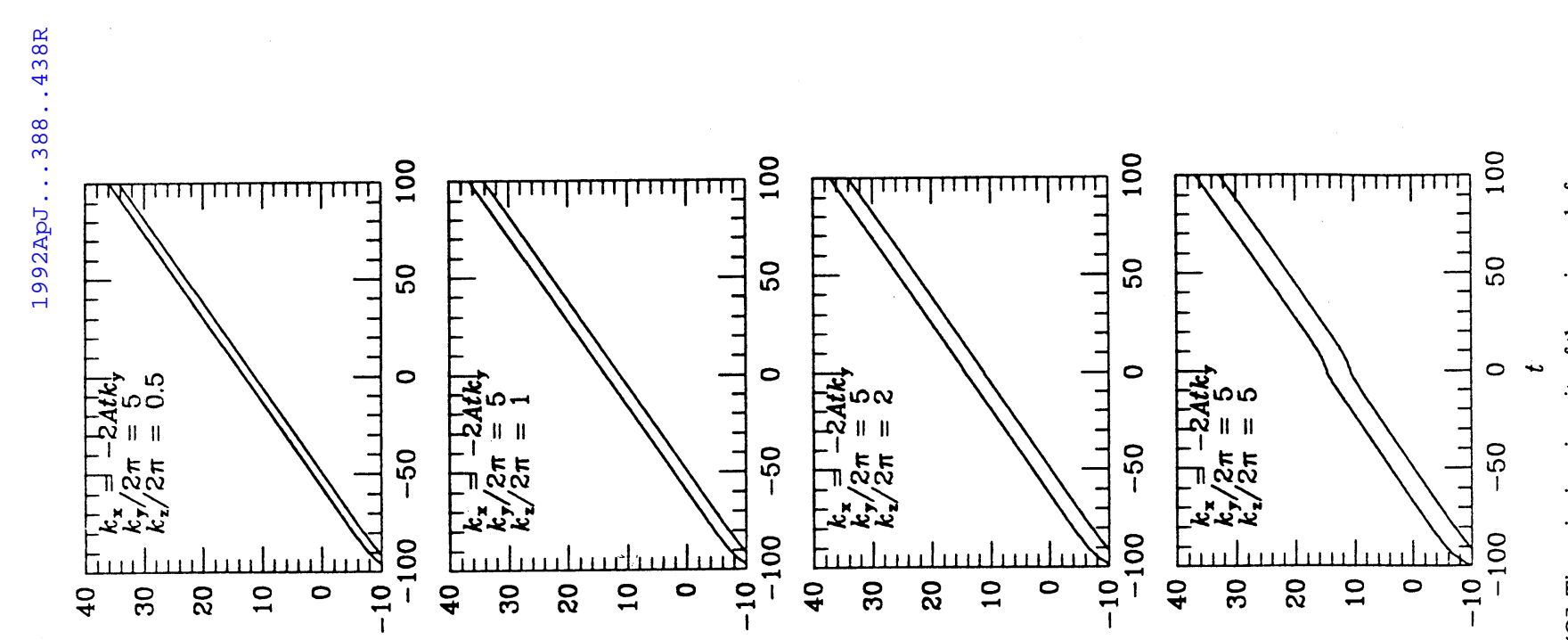

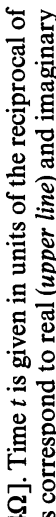

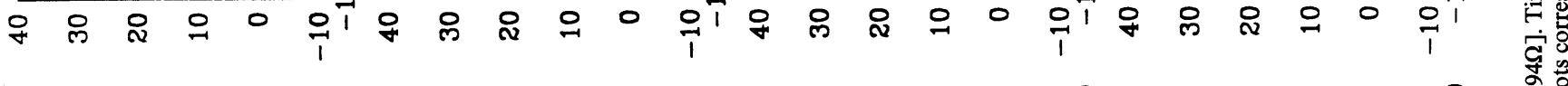
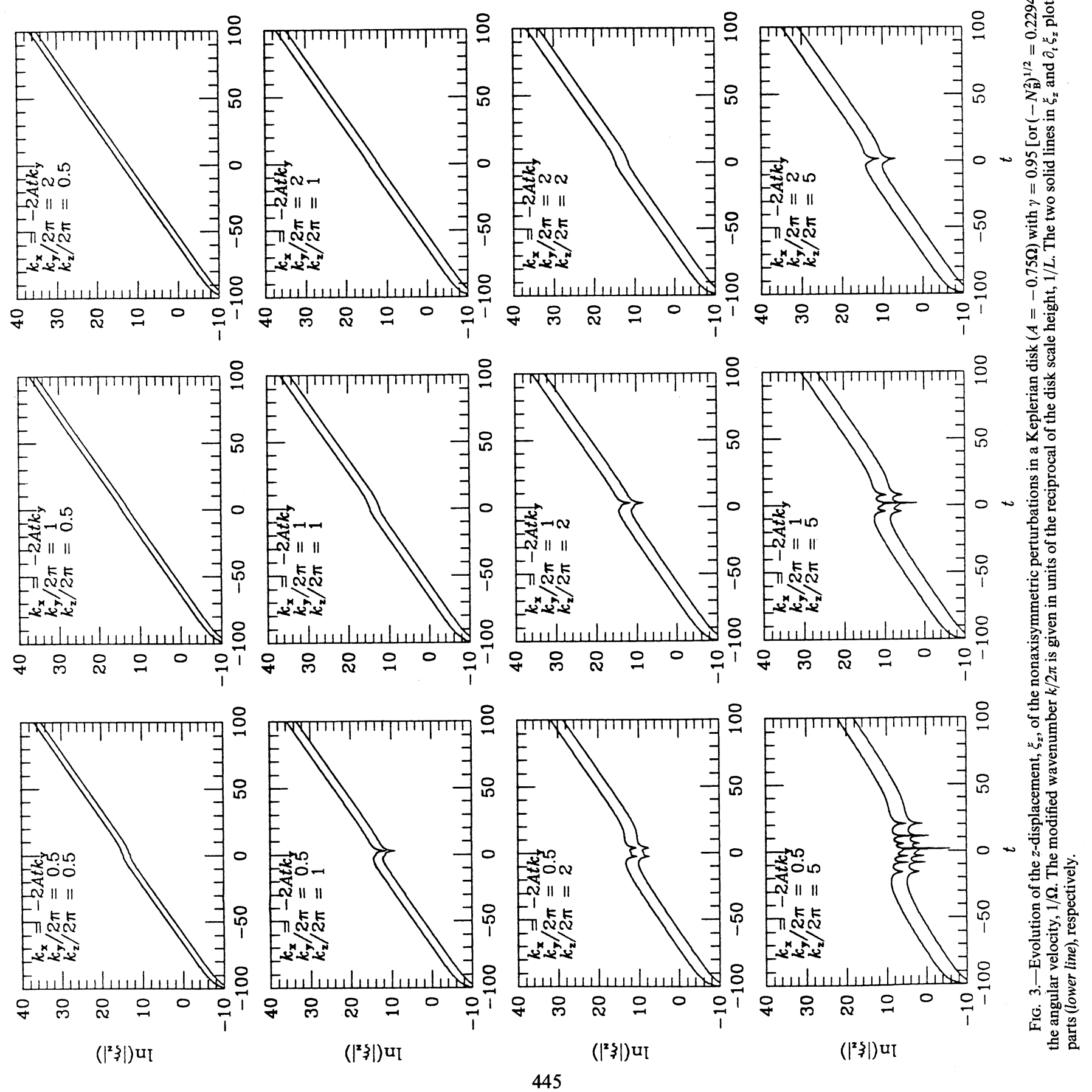

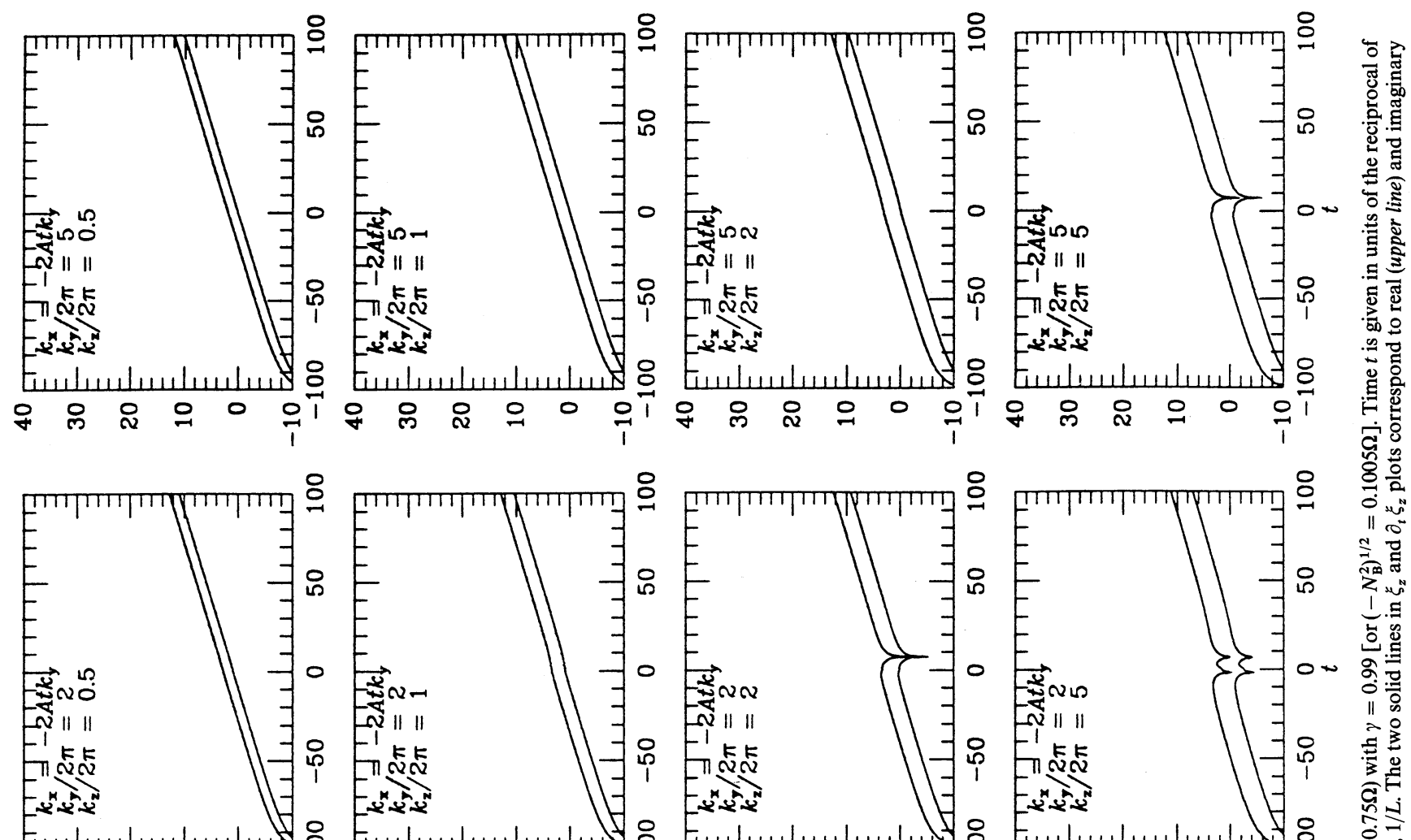

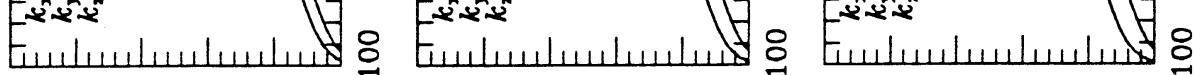

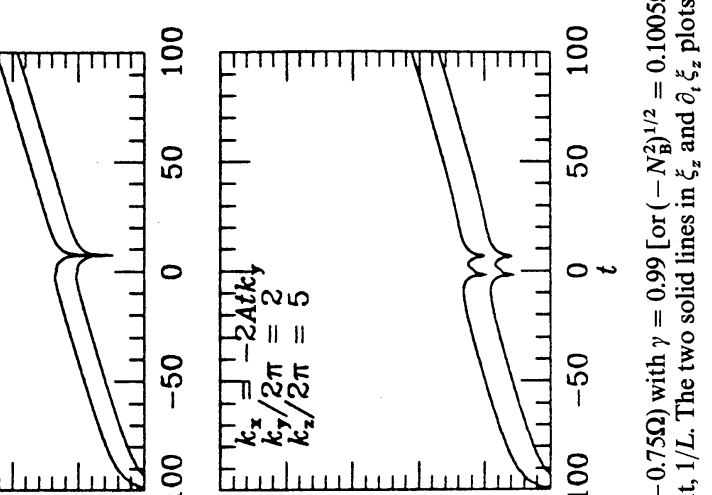

:

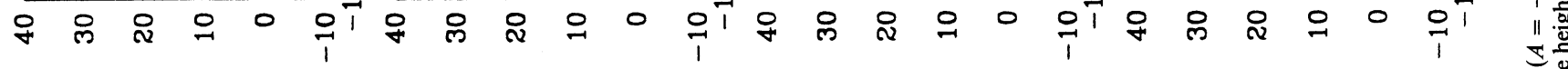

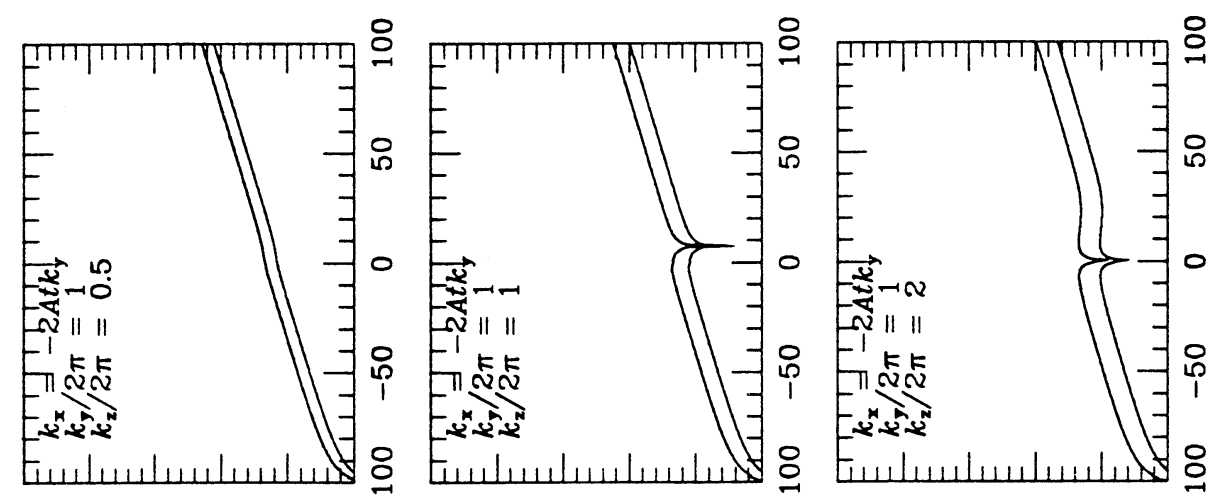

o $\begin{aligned} & 0 \\ & 0\end{aligned}$
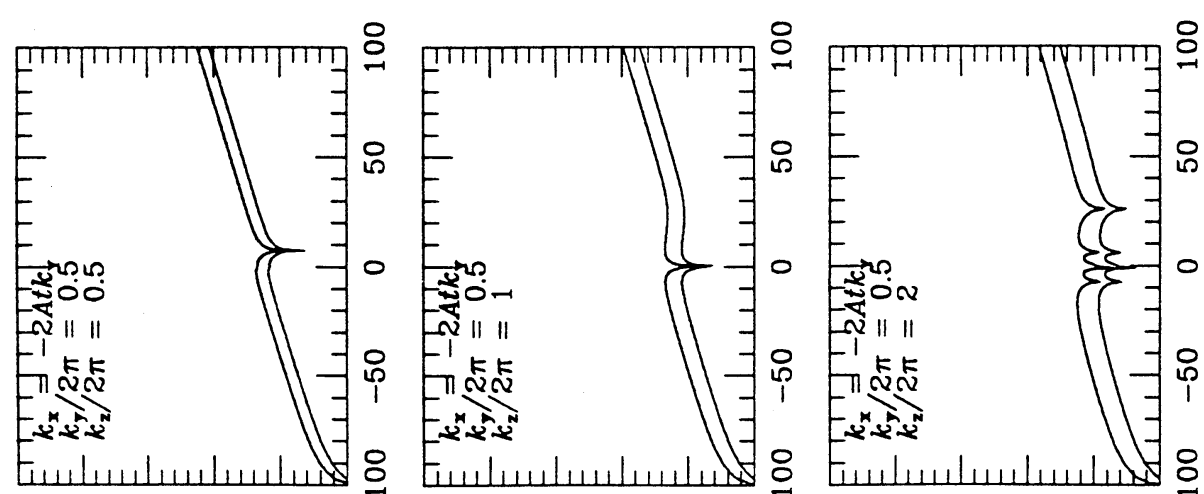

o $\begin{aligned} & 8 \\ & 0\end{aligned}$

$\left(||^{\xi} \mid\right)$ uा

$\left(||^{2} \mid\right)$ uा

$\left(||^{2} \mid\right)$ uा

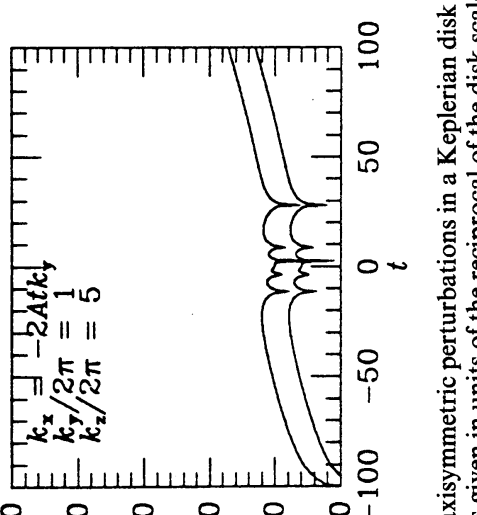

일.

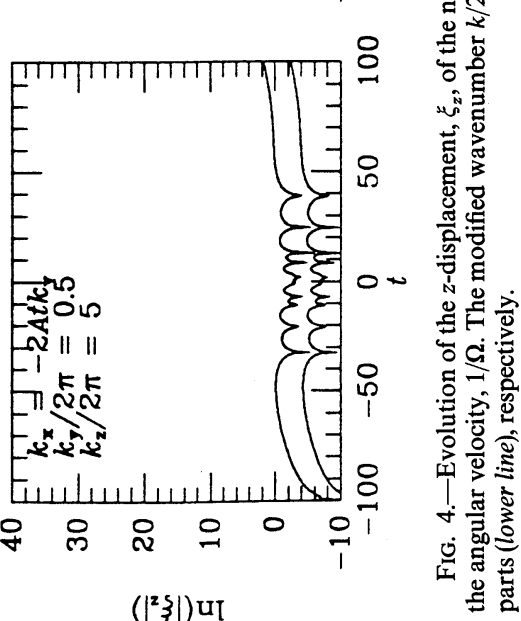


TABLE 2

Average Growth Rate in UNits of $\Omega$ Between $-10 / \Omega \leq t \leq 10 / \Omega$ IN A Keplerian DisK ${ }^{a}$

\begin{tabular}{|c|c|c|c|c|c|c|}
\hline & $k_{y} / 2 \pi=0.2$ & $k_{y} / 2 \pi=0.5$ & $k_{y} / 2 \pi=1.0$ & $k_{y} / 2 \pi=2.0$ & $k_{y} / 2 \pi=5.0$ & $k_{y} / 2 \pi=10$. \\
\hline \multicolumn{7}{|c|}{$\gamma=0.95\left[\left(-N_{\mathrm{B}}^{2}\right)^{1 / 2}=0.2294 \Omega\right]$} \\
\hline$k_{z} / 2 \pi=0.2 \ldots$ & 0.1271 & 0.1876 & 0.2166 & 0.2266 & 0.2290 & 0.2293 \\
\hline$k_{z} / 2 \pi=0.5 \ldots$ & 0.0301 & 0.1344 & 0.1818 & 0.2108 & 0.2270 & 0.2288 \\
\hline$k_{z} / 2 \pi=1.0 \ldots$ & 0.0000 & 0.0597 & 0.1353 & 0.1823 & 0.2189 & 0.2270 \\
\hline$k_{z} / 2 \pi=2.0 \ldots \ldots \ldots$ & 0.0000 & 0.0000 & 0.0600 & 0.1355 & 0.1926 & 0.2190 \\
\hline$k_{z} / 2 \pi=5.0 \ldots \ldots \ldots$ & 0.0000 & 0.0000 & 0.0000 & 0.0315 & 0.1355 & 0.1825 \\
\hline$k_{z} / 2 \pi=10 \ldots \ldots \ldots$ & 0.0000 & 0.0000 & 0.0000 & 0.0000 & 0.0601 & 0.1356 \\
\hline \multicolumn{7}{|c|}{$\gamma=0.99\left[\left(-N_{\mathrm{B}}^{2}\right)^{1 / 2}=0.1005 \Omega\right]$} \\
\hline$k_{z} / 2 \pi=0.2 .$. & 0.0147 & 0.0603 & 0.0805 & 0.0929 & 0.0995 & 0.1003 \\
\hline$k_{z} / 2 \pi=0.5 \ldots \ldots \ldots$ & 0.0000 & 0.0183 & 0.0539 & 0.0759 & 0.0936 & 0.0992 \\
\hline$k_{z} / 2 \pi=1.0 \ldots \ldots \ldots$ & 0.0000 & 0.0000 & 0.0187 & 0.0542 & 0.0816 & 0.0938 \\
\hline$k_{z} / 2 \pi=2.0 \ldots \ldots \ldots$ & 0.0000 & 0.0000 & 0.0000 & 0.0188 & 0.0624 & 0.0817 \\
\hline$k_{z} / 2 \pi=5.0 \ldots \ldots \ldots$ & 0.0000 & 0.0000 & 0.0000 & 0.0000 & 0.0189 & 0.0543 \\
\hline$k, / 2 \pi=10 \ldots \ldots \ldots$ & 0.0000 & 0.0000 & 0.0000 & 0.0000 & 0.0000 & 0.0189 \\
\hline
\end{tabular}

a Estimated from the approximate dispersion relation, see text for details.

RPL have pointed out that thermal conduction by radiation is likely to be a significant effect in realistic disks and that it suppresses the growth of axisymmetric modes at sufficiently large wavenumbers. Hence it seems likely that there exist completely stable equilibria with $N_{\mathbf{B}}^{2}<0$ and $\kappa^{2}>0$ if the conductivity is sufficiently large. In other words, unless radiative conduction is negligible a Keplerian disk must be more than marginally superadiabatic before convection will develop. We further expect that the growth of nonaxisymmetric modes should eventually cease at sufficiently large $\left|k_{x}\right|$ in the presence of thermal conduction; hence in principle nonaxisymmetric disturbances should experience only a finite amplification factor, as is the case for nonaxisymmetric density waves in the shearing sheet (cf. Toomre 1981). We may expect that this amplification factor should depend exponentially upon the reciprocal of the conductivity.

Unfortunately, we cannot demonstrate the effects of thermal conduction in our $\gamma<1$ constant scale-height model. Although it is a simple matter to add a conduction term, $\nabla \cdot[K \rho \nabla(p / \rho)]$, where $K$ is the conductivity to the right-hand side of the energy equation $(2.5 \mathrm{c})$, it turns out that for $\gamma<1$ the conductivity introduces spurious instabilities that have nothing to do with convection. With one sign of the conductivity, isobaric temperature fluctuations are destabilized, even in the absence of a gravitational field; and with the other sign, there appear isothermal sound waves, with propagation speed $c_{\text {iso }} \equiv c_{s} /$ $\gamma^{1 / 2}>c_{s}$, that grow at a rate inversely proportional to the conductivity(!). ${ }^{3}$ Some such pathology might have been expected in our $\gamma<1$ model, since as we pointed out in $\S 2$, they are hydrodynamically but not thermodynamically reasonable.

A primary motivation for studying convective modes in differentially rotating disks is, of course, to explore the viability of convection as a mechanism for angular momentum transport. We shall not attempt to estimate the viscosity parameter $\alpha$ on the basis of our linearized calculations. It is, however, appro-

\footnotetext{
${ }^{3}$ Similar waves appear for $\gamma>1$, but they damp rather than grow at rate $\propto\left(c_{s}^{2}-c_{\text {iso }}^{2}\right) / K$. Even here the reciprocal dependence on $K$ seems paradoxical, but note that these waves do not exist at all for $K=0$.
}

priate to ask what radial flux of angular momentum one of our modes will sustain at a given (quasi)linear amplitude. As we have noted in $\S 1$ and will see explicitly below, axisymmetric modes produce no angular-momentum flux.

As is well known, linear waves on stationary axisymmetric backgrounds are associated with energy and angular momentum densities, and corresponding fluxes, which are quadratic in the wave amplitude. These densities and fluxes are defined as averages over a wavelength. In the absence of dissipation, the density $\rho$ and flux $j$ satisfy a continuity equation:

$$
\partial_{t} \rho+\nabla \cdot j=0
$$

Since our Fourier modes have spatially constant average mean-square amplitudes, $\boldsymbol{j}$ is constant for them and hence the modes produce no change in the angular momentum density, i.e., $\partial_{t} \rho_{J}=0$. However, $\partial_{t} \rho_{J}$ could be nonzero for a localized wavepoint consisting of a superposition of such modes, provided $\boldsymbol{j}$ does not vanish for all of these modes. We are here mainly concerned with the radial component of the angular momentum flux, which we denote simply by $j$.

An expression for $j$ in terms of the Lagrangian displacements may be derived by applying Noether's theorem to the secondorder Lagrangian density (cf. Narayan, Goldreich, \& Goodman 1987, Appendix A),

$$
\begin{aligned}
\mathscr{L}_{2} \equiv \frac{1}{2} \rho^{(o)}\left\{\left|D_{t} \xi\right|^{2}-\right. & \frac{p^{(o)}}{\rho^{(o)}}\left[(\gamma-1)(\nabla \cdot \xi)^{2}+\partial_{i} \xi_{j} \partial_{j} \xi_{i}\right] \\
& \left.+2 \epsilon_{i j k} \xi_{i} D_{t} \xi_{j} \Omega_{k}-2 \xi_{i} \xi_{j} \partial_{i} \partial_{j} \Phi\right\} .
\end{aligned}
$$

Here $\rho^{(o)}$ and $p^{(o)}$ are the unperturbed mass density and pressure and $\Phi=2 A \Omega x^{2}+g|z|$ is the external gravitational potential. The second-order hydrodynamic action is obtained by integrating $\mathscr{L}_{2}$ over time and over volume in Lagrangian coordinates $d^{3} r^{(o)}$ (i.e., $r^{(o)}$ is the unperturbed position of the fluid element at a given time). The Euler-Lagrange equations that follow by requiring this action to be stationary under variations in $\xi$ are the linearized equations of motion (2.18). 
Noether's theorem then gives

$$
\begin{aligned}
j & =-\frac{\partial \mathscr{L}_{2}}{\partial\left(\partial_{x} \xi_{i}\right)} \partial_{y} \xi_{i} \\
& =p^{(o)}\left[\gamma(\nabla \cdot \xi) \partial_{y} \xi_{x}+\left(\partial_{z} \xi_{x} \partial_{y} \xi_{z}-\partial_{y} \xi_{x} \partial_{z} \xi_{z}\right)\right] .
\end{aligned}
$$

Note the minus sign appearing on the right-hand side of equation (4.3).

As sophisticated readers will know, the physical interpretation of second-order wave fluxes is fraught with subtleties and the following remarks are to allay any consequent worries: (i) The flux (4.3) is a function of position in Lagrangian coordinates, $\left\{\boldsymbol{r}^{(\boldsymbol{o})}\right\}$, which follow the fluid, so $j$ as defined represents a flux of angular momentum from one fluid element to another, it is not contaminated by any angular-momentum advection due to a possible radial mass flux. (ii) Narayan, et al. (1987) demonstrate that the angular-momentum density associated with this $j$ is truly the second-order part of the total physical angular momentum of the fluid per unit Lagrangian volume, notwithstanding any possible decomposition of $j$ into parts attributable separately to the wave and to a change in the background flow. (Actually, their demonstration is given for two dimensions only, but it generalizes easily to three.)

As derived, $j$ has the dimensions of a linear momentum flux rather than an angular momentum flux, because we work with the pseudo-Cartesian azimuthal coordinate $y$ rather than the angle $\theta$ (cf. eq. [2.3b]); the actual angular momentum flux is $j$ times the reference radius $r_{0}$. One sees explicitly from equation (4.3) that the angular-momentum flux of axisymmetric modes vanishes, since $\partial_{y} \xi=0$.

Although the form (4.3) for $j$ is simple, it is instructive to use the equations (2.23) to reexpress $j$ in terms of a single fluid variable, say $\xi_{x}$. For this purpose, we have used $\xi$ of the form

$$
\begin{aligned}
\xi_{x}(x, y, z, t)=X_{x} \exp (s t) & \cos \left(k_{x} x+k_{y} y\right) \\
& \times \cos \left(k_{z} z-\theta\right) \exp \left(\frac{z}{2 L}\right),
\end{aligned}
$$

$\xi_{y}(x, y, z, t)=X_{y} \exp (s t) \cos \left(k_{x} x+k_{y} y\right)$

$$
\times \cos \left(k_{z} z-\theta\right) \exp \left(\frac{z}{2 L}\right),
$$

$\xi_{z}(x, y, z, t)=-X_{z} \exp (s t) \sin \left(k_{x} x+k_{y} y\right)$

$$
\times \sin \left(k_{z} z\right) \exp \left(\frac{z}{2 L}\right),
$$

where $\theta$ is the phase angle between $\xi_{x}$ (or $\xi_{y}$ ) and $\xi_{z}$ and proceded as though $s$ and $k_{x}$ were constant, which is justified at least for $\left|k_{x}(t)\right| \gg\left|k_{y}\right|$. Note that the above form of $\xi$ satisfies explicitly the boundary condition in equation (2.25). Then, we obtain

$$
j=-\frac{1}{4} \rho(0)\left|\xi_{x}(t)\right|^{2} k_{y} \frac{s\left(s^{2}+\kappa^{2}\right)}{s k_{x}+2 \Omega k_{y}},
$$

where $\xi_{x}(t)=X_{x} \exp (s t)$.

Since the nonaxisymmetric modes grow as they shear, we expect their amplitudes to be largest at strongly trailing wavenumbers $\left|k_{x}(t)\right| \gg\left|k_{y}\right|$ and $k_{x}(t) k_{y}>0$. On the other hand, for growing modes $s>0$. It then follows from equation (4.5) that $j<0$; in other words, the convectively unstable modes eventually transport angular momentum inwards rather than

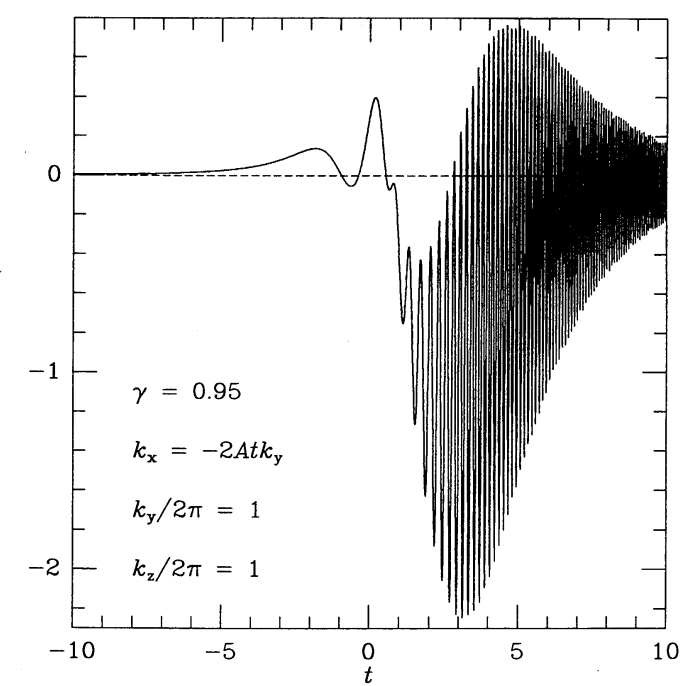

Fig. 5.-Evolution of the angular momentum flux $j$, normalized by $\rho^{(o)}\left(\delta v_{x}^{2}\right.$ $\left.+\delta v_{y}^{2}+\delta v_{z}^{2}\right)$, for nonaxisymmetric perturbations in a Keplerian disk $(A=-0.75 \Omega)$ with $\gamma=0.95\left[\left(-N_{\mathrm{B}}^{2}\right)=0.2294 \Omega\right]$. Here, $\delta v_{x}, \delta v_{y}$, and $\delta v_{z}$ are the Eulerian velocity perturbations. Vertical and azimuthal wavelengths are equal to the disk scale height, $L$. Time $t$ is given in units of the reciprocal of the angular velocity, $1 / \Omega$.

outward. In a disk of finite radial width these modes would therefore be expected to reinforce rather than resist the differential rotation.

Figure 5 shows the evolution of the angular momentum flux $j$ evaluated using equation (4.3) and normalized by $\rho^{(o)}\left(\delta v_{x}^{2}\right.$ $\left.+\delta v_{y}^{2}+\delta v_{z}^{2}\right)$ for nonaxisymmetric perturbations in $\mathbf{a}$ Keplerian disk $(A=-0.75 \Omega)$ with $\gamma=0.95\left[\left(-N_{\mathbf{B}}^{2}\right)^{1 / 2}=\right.$ $0.2294 \Omega$ ] and with the azimuthal and vertical wavenumbers $k_{y}=k_{z}=2 \pi / L$. (This is the same case as considered in Fig. 2.) Here, $\delta v_{x}, \delta v_{y}$, and $\delta v_{z}$ are the Eulerian velocity perturbations which are expressed in terms of the displacements as

$$
\begin{gathered}
\delta v_{x}=\partial_{t} \xi_{x}, \\
\delta v_{y}=\partial_{t} \xi_{y}-2 A \xi_{x}, \\
\delta v_{z}=\partial_{t} \xi_{z}
\end{gathered}
$$

At $t<0$ or $\left[k_{x}(t) / k_{y}<0\right], j$ remains positive until $\left|k_{x}(t)\right| \sim\left|k_{y}\right|$. On the other hand, at $t>0$ or $\left[k_{x}(t) / k_{y}>0\right], j$ oscillated with a frequency corresponding to the sound wave frequency with the instantaneous wavenumber $\boldsymbol{k}(t) \equiv\left[k_{x}(t), k_{y}, k_{z}\right]$, but on average $j<0$. As $t \rightarrow+\infty$ or $\left[k_{x}(t) / k_{y} \rightarrow+\infty\right]$, the oscillation frequency becomes infinite and the oscillation amplitude becomes zero and $j$ stays monotonically negative, which agrees with the result of the last paragraph.

What holds for the linear modes need not be true for nonlinear, turbulent convection. Nevertheless, the result seems paradoxical. But one should remember that the free energy for these modes does not come directly from the shear but rather from the unstable vertical stratification, so the modes are not obliged to resist the shear. Of course, in a self-consistent model for the effective viscosity, the nonlinear convection is required somehow to tap the energy in the shear, and the kinetic energy of the convection is required to be dissipated in such a way as to reinforce the superadiabatic vertical stratification. So perhaps this paradox merely demonstrates the hazards extrapolating from linear modes into nonlinear regimes. 
On the other hand, suppose that the nonlinear convection forms fairly steady, subsonic, axisymmetric rolls spanning the disk thickness and braked by small-scale turbulent viscosity (a picture that RPL may have had in mind when they estimated $\alpha$ from their axisymmetric modes). Where neighboring rolls touch, the one centered at a larger radius will have the larger angular momentum per unit mass and hence the larger angular velocity. Hence if small-scale turbulent viscosity produces friction between these rolls, angular momentum will be transferred from the roll at larger radius to the inner one. Perhaps convection, being a mixing process, attempts to equalize not the angular velocity $(\Omega)$ but the angular momentum per unit mass $\left(\Omega r^{2}\right)$ (Biermann 1951).

This work was in part supported by David Lucille Packard Foundation Fellowship and by a grant from NASA's Astrophysics Theory Program.

\section{APPENDIX A \\ EIGENMODES}

The form of the eigenfunction assumed in equation (2.22) has been used previously in the context of the stability analysis of an exponentially stratified atmosphere (see, e.g., Chandrasekhar 1961). But, in the discussion of the Parker instability, Lerche \& Parker (1967) (and also Shu [1974] for three-dimensional perturbations in a rigidly rotating disk) assumed a different spatial dependence of the exponential factor. While the choice in the equation (2.22) may be justified in several different ways, we will use the formalism used in Bernstein et al. (1958) and Frieman \& Rotenberg (1960) to justify the choice for the two limiting cases considered in $\S 3.1$ and 3.2 , the case of a rigidly rotating disk $(A=0)$ and the case of axisymmetric modes $\left(k_{y}=0\right)$. In those cases, true normal mode solutions exist with the $\xi$ time-dependence of the form exp $(i \omega t)$ and the mathematical manipulation becomes simpler. However, the same justification could be made for general cases with $A \neq 0$ and $k_{y} \neq 0$.

Let us consider $\xi$ of the form

$$
\xi\left(x^{\prime}, y^{\prime}, z^{\prime}, t\right)=\xi \exp (i \omega t) \exp \left(\frac{\left|z^{\prime}\right|}{2 L}+\mu z^{\prime}\right) \exp \left(i k_{x} x^{\prime}+i k_{y} y^{\prime}+i k_{z} z^{\prime}\right) .
$$

The perturbation equations can be written in a compact form

$$
\begin{gathered}
-\omega^{2} \rho \xi+2 i \omega \rho \Omega \hat{z} \times \xi-\boldsymbol{F}(\xi)=0, \\
\boldsymbol{F}(\xi)=-4 A \Omega \xi_{x} \hat{\boldsymbol{x}}+c_{s}^{2} \nabla(\rho \nabla \cdot \xi)-\rho \nabla\left(g \xi_{z}\right)+g \rho \nabla \cdot \xi \hat{z} \\
=-4 A \Omega \xi_{x} \hat{\boldsymbol{x}}-c_{s}^{2} \rho \boldsymbol{k}_{T}\left(\boldsymbol{k}_{T} \cdot \xi\right)-i c_{s}^{2} \rho \frac{\boldsymbol{k}_{T}}{L} \cdot \xi \hat{z}-i g \rho \boldsymbol{k}_{T} \xi_{z}+i g \rho \boldsymbol{k}_{T} \cdot \xi \hat{z},
\end{gathered}
$$

where

$$
\boldsymbol{k}_{T} \equiv k_{x} \hat{\boldsymbol{x}}+k_{y} \boldsymbol{y}+\left(k_{z}-\frac{i}{2 L}-i \mu\right) \hat{z} .
$$

The self-adjointness of $\boldsymbol{F}$ states that, for any two displacement vectors $\boldsymbol{\xi}$ and $\zeta$ in a finitely extended fluid,

$$
\int[\boldsymbol{F}(\xi) \cdot \zeta-F(\zeta) \cdot \xi] d^{3} x=0 .
$$

This property can be proved by converting the volume integration into the surface integration and using the appropriate boundary conditions, e.g., a fluid-fluid interface or a fluid-vacuum interface (see, for instance, Kulsrud 1964). On the other hand, from $F$ in the equation (A2b), we have

$$
\begin{aligned}
\int[\boldsymbol{F}(\xi) & \left.\cdot \xi^{*}-\boldsymbol{F}^{*}(\xi) \cdot \xi\right] d^{3} x=-2 i c_{s}^{2} \mu \int d^{3} x \rho\left[k_{x}\left(\xi_{x} \xi_{z}^{*}+\xi_{x}^{*} \xi_{z}\right)+k_{y}\left(\xi_{y} \xi_{z}^{*}+\xi_{y}^{*} \xi_{z}\right)+2 k_{z} \xi_{z} \xi_{z}^{*}\right] \\
& =2 c_{s}^{2} \mu \frac{\left[c_{s}^{2}(1 / 2 L+\mu)-g\right] k_{h}^{2}\left(\omega^{2}-\omega^{* 2}\right)-2 i k_{z}\left[|\omega|^{4}-\left(\omega^{2}+\omega^{* 2}\right)\left(\kappa^{2}+c_{s}^{2} k_{h}^{2} / 2\right)+\kappa^{2}\left(\kappa^{2}+c_{s}^{2} k_{h}^{2}\right)\right]}{\left|\omega^{2}-c_{s}^{2} k_{h}^{2}-\kappa^{2}\right|^{2}} \int d^{3} x \rho \xi_{z} \xi_{z}^{*}=0,
\end{aligned}
$$

where $k_{h}^{2} \equiv k_{x}^{2}+k_{y}^{2}$ and $\kappa^{2} \equiv 4 \Omega(A+\Omega)$. This requires, in general, $\mu=0$ and the eigenfunction in equation (A1) reduces into the one in equation (2.22).

\section{APPENDIX B}

\section{OVERSTABILITY}

We prove that $\omega^{2}$ (or $-s^{2}$ ) is real in the two limiting cases considered in $\S 3$, the case of a rigidly rotating disk $(A=0)$ and the case of axisymmetric modes $\left(k_{y}=0\right)$. We follow the method used in Zweibel \& Kulsrud (1975). From the perturbation equation in 
equation (A2), we get

$$
\left(\omega^{2}-\omega^{* 2}\right) \int \rho \xi \cdot \xi^{*} d^{3} x-2 i\left(\omega-\omega^{*}\right) \int \rho \Omega \cdot\left(\xi \times \xi^{*}\right) d^{3} x=0,
$$

where the self-adjointness of $\boldsymbol{F}$ has been used. Using the relation between $\xi_{x}$ and $\xi_{y}$ again from the perturbation equation in (2.23)

$$
\xi_{y}=\frac{\omega k_{y}+2 i \Omega k_{x}}{\omega k_{x}-2 i \Omega k_{y}} \xi_{x}
$$

the above equation becomes

$$
\left(\omega^{2}-\omega^{* 2}\right)\left[\frac{|\omega|^{2}\left(k_{x}^{2}+k_{y}^{2}\right)}{\left|\omega k_{x}-2 i \Omega k_{y}\right|^{2}} \int \rho \xi_{x} \xi_{x}^{*} d^{3} x+\int \rho \xi_{z} \xi_{z}^{*} d^{3} x\right]=0 .
$$

Since the factor in bracket is positive definite, $\omega^{2}-\omega^{* 2}$ must be equal to zero, hence $\omega^{2}$ is real. Thus, in the cases with $A=0$ or $k_{y}=0$, overstability cannot occur.

\section{REFERENCES}

Balbus, S. A., \& Hawley, J. F. 1991, ApJ, 376, 214 Bernstein, I. B., Frieman, E. A., Kruskal, M. D., \& Kulsrud, R. M. 1958, Proc.
R. Soc. Lond. A, 244, 17

Biermann, L. 1951, Z. Astrophys., 28, 304

Cameron, A. G. W. 1978a, in The Origin of the Solar System, ed. S. F. Dermott, (Chichester: Wiley), 49

1978b, Moon, 18, 5

Chandrasekhar, S. 1961, Hydrodynamic and Hydromagnetic Stability (London: Oxford Univ.)

Frieman, E. A., \& Rotenberg, M. 1960, Rev. Mod. Phys., 32, 898

Goldreich, P., \& Lynden-Bell, D. 1965, MNRAS, 130, 125

Hawley, J. F., \& Balbus, S. A. 1991, ApJ, 376, 223

Julian, W. H., \& Toomre, A. 1966, ApJ, 146, 810

Kulsrud, R. M. 1964, in Proc. International School of Physics, Course 25:

Advanced Plasma Theory, ed. M. N. Rosenbluth (New York: Academic), 54
Lerche, I., \& Parker, E. N. 1967, ApJ, 149, 559

Lin, D. N. C., \& Papaloizou, J. C. B. 1980, MNRAS, 191, 37

Lynden-Bell, D., \& Pringle, J. E. 1974, MNRAS, 168, 603

Mihalas, D., \& Binney, J: 1981, Galactic Astronomy (San Francisco: Freeman)

Narayan, R., Goldreich, P., \& Goodman, J. 1987, MNRAS, 228, 1

Parker, E. N. 1966, ApJ, 145, 811

Pringle, J. E. 1981, ARA\&A, 19, 137

Ruden, S. P., Papaloizou, J. C. B., \& Lin, D. N. C. 1988, ApJ, 329, 739 (RPL)

Shakura, N. J., \& Sunyaev, R. A. 1983, A\&A, 24, 337

Shu, F. H. 1974, A\&A, 33, 55

Toomre, A. 1981, in The Structure and Evolution of Normal Galaxies, ed

S. M. Fall \& D. Lynden-Bell (London: Cambridge Univ. Press), 111

Zweibel, W. G., \& Kulsrud, R. M. 1975, ApJ, 201, 63 